\title{
AlGaInP red-emitting light emitting diode under extremely high pulsed pumping
}

\author{
Amit Yadav*a, Ilya E. Titkov ${ }^{a}$, Grigorii S. Sokolovskii ${ }^{b}$, Sergey Yu. Karpovc, Vladislav V. Dudelev ${ }^{\mathrm{b}}$, \\ Ksenya K. Soboleva ${ }^{\mathrm{d}}$, Martin Strassburg ${ }^{\mathrm{e}}$, Ines Pietzonka ${ }^{\mathrm{e}}$, Hans-Juergen Lugauer ${ }^{\mathrm{e}}$, Edik U. Rafailov ${ }^{\mathrm{a}}$ \\ ${ }^{\mathrm{a} O p t o e l e c t r o n i c s ~ a n d ~ B i o m e d i c a l ~ P h o t o n i c s ~ G r o u p, ~ A s t o n ~ I n s t i t u t e ~ o f ~ P h o t o n i c ~ T e c h n o l o g i e s, ~ A s t o n ~}$ \\ University, Birmingham, B4 7ET, UK \\ ${ }^{b}$ Ioffe Physico-Technical Institute, Politekhnicheskaya str. 26, 194021 St. Petersburg, Russia

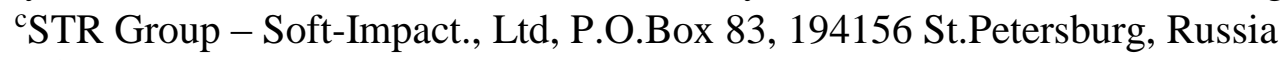 \\ ${ }^{\mathrm{d} S a i n t-P e t e r s b u r g ~ S t a t e ~ P o l y t e c h n i c a l ~ U n i v e r s i t y, ~ S t . ~ P e t e r s b u r g, ~ R u s s i a ~}$ \\ eOSRAM Opto Semiconductors GmbH, CTO AdvancedConcepts \& Engineering, Novel \\ Technologies, Leibnitzstr., 93055 Regensburg, Germany
}

\begin{abstract}
Efficiency of commercial $620 \mathrm{~nm}$ AlGaInP Golden Dragon-cased high-power LEDs has been studied under extremely high pump current density up to $4.5 \mathrm{kA} / \mathrm{cm}^{2}$ and pulse duration from microsecond down to sub-nanosecond range. To understand the nature of LED efficiency decrease with current, pulse width variation is used. Analysis of the pulse-duration dependence of the LED efficiency and emission spectrum suggests the active region overheating to be the major factor controlling the LED efficiency reduction at $\mathrm{CW}$ and sub-microsecond pumping. The overheating can be effectively avoided by the use of sub-nanosecond current pulses. A direct correlation between the onset of the efficiency decrease and LED overheating is demonstrated.
\end{abstract}

Keywords: AlGaInP LEDs, external quantum efficiency, self-heating, efficiency droop

\section{INTRODUCTION}

Light emitting diodes (LEDs) based on AlGaInN and AlGaInP materials systems cover the whole visible spectrum from 400 to $700 \mathrm{~nm}$. While the AlGaInN based LEDs are most efficient in the blue-green spectral region, the long-wavelength region of the visible spectrum around yellow to red is benefitted by the efficient emission from the AlGaInP LEDs. The LEDs utilizing $\left(\mathrm{Al}_{x} \mathrm{Ga}_{1-x}\right)_{0.5} \mathrm{In}_{0.5} \mathrm{P}$ quaternary alloys are normally lattice matched to their GaAs substrate of growth. These materials can provide light emission at the wavelengths from $560 \mathrm{~nm}$ to $650 \mathrm{~nm}$ by varying the aluminium (Al) content until the band gap becomes indirect around $555 \mathrm{~nm}(x \sim 0.53-0.56)^{1}$. The application area for these devices ranges from indicator lamps to outdoor and automotive lighting ${ }^{2}$. Though specific applications influence requirements on the device parameters, LEDs with higher efficiency over broad range of operating conditions are always beneficial and desirable.

It has been commonly observed that external quantum efficiency (EQE) of both AlGaInP and AlGaInN emitters, decreases with the forward current at high current densities ${ }^{3}$. This decrease of the efficiency limits LEDs to achieve their utmost performance and is being investigated intensively. In case of AlGaInN LEDs, the proposed dominant mechanisms responsible for the efficiency decrease (droop) are: (a) carrier leakage either due to asymmetric junction or polarization or both $^{4,5}$, (b) saturated radiative recombination ${ }^{6}$, (c) Auger recombination ${ }^{7,8}$, (d) delocalization of carriers ${ }^{9}$ and (e) dislocation-induced non-radiative recombination. ${ }^{10}$ Observed similarities in EQE vs. current behaviour of AlGaInP and $\mathrm{AlGaInN}$ LEDs points to a possible common mechanism leading to the efficiency decrease for both material systems ${ }^{11}$. However, for AlGaInP devices, polarization-induced electron leakage, increased non-radiative recombination at dislocations, and carrier delocalization are unlikely to dominate because of the absence of strong piezoelectric fields, the

Light-Emitting Diodes: Materials, Devices, and Applications for Solid State Lighting XX, edited by Heonsu Jeon,

Li-Wei Tu, Michael R. Krames, Martin Strassburg, Proc. of SPIE Vol. 9768, 97681K · @ 2016 SPIE

CCC code: $0277-786 \mathrm{X} / 16 / \$ 18 \cdot$ doi: $10.1117 / 12.2213344$ 
use of heterostructures lattice-matched to GaAs substrate, and much weaker tendency of the alloy to carrier localization due to composition fluctuation, respectively.

At low current densities, carrier leakage due to asymmetric p-n junction has been suggested to contribute towards efficiency decrease in AlGaInP LEDs ${ }^{12,13}$. Comprehensive studies of internal quantum efficiency (IQE) of amber/red AlGaInP LEDs $^{14,15}$ have attributed the leakage to insufficiently high barriers separating quantum wells (QWs) in the LED active regions. They found the leakage to depend strongly on temperature with the activation energy nearly equal to the difference between the electron energy levels in the QWs and the conduction band bottom in the barriers. From this point of view, pushing up the electron quasi-Fermi level due to increase of the electron concentration in the QWs and device self-heating should produce similar effects on the efficiency reduction. It should be noted that elevated operating temperature may lower the LED efficiency by itself without enhancing the electron leakage. Indeed, the radiative recombination constant generally decreases with temperature, whereas the non-radiative carrier lifetime becomes shorter, i.e. both factors reduce $\mathrm{IQE}$ of the active region material. In order to distinguish between the roles of the MQW barrier heights controlling the electron leakage and elevated temperature by itself in operation of amber/red LEDs, special experiments are required, including LED examination under short-pulse current injection and rather high current densities. To our knowledge, such experiments have not yet been reported before.

The effect of device overheating and efficiency thermal rollover in LEDs can be mitigated by a packaged heat sink and/or by driving the device with short pulses with the duty cycle $\leq 1 \%{ }^{16}$. In this paper, we will compare the behaviour of EQE in commercial $620 \mathrm{~nm}$ red high-power AlGaInP LED under continuous wave (CW) and microsecond-to-sub-nanosecond current-pulse excitation in the width modulation (PWM) regime up to the current densities of $\sim 4.5 \mathrm{kA} / \mathrm{cm}^{2}$. Detailed characterization of the device under CW and PWM pumping is important for making conclusion on the nature of the efficiency reduction with current in phosphide LEDs.

\section{DEVICE STRUCTURE}

The $1 \mathrm{~mm} \times 1 \mathrm{~mm}$ high power red LEDs based on AlGaInP (Fig.1) used in this study have multiple quantum well (MQW) active regions emitting at $620 \mathrm{~nm}$ at room temperature. $\mathrm{Al}_{0.5} \mathrm{In}_{0.5} \mathrm{P}$ layers are used for confining the carriers with an $\mathrm{AlGaAs}$ window and p-GaAs contact layer. The structure was grown on GaAs substrate. OSRAM OS's standard Thinfilm process has been applied for processing the devices and their packaging in Golden Dragon cases.

\begin{tabular}{|c|}
\hline p- cap GaAs \\
\hline $\mathrm{p}$-contact layer $\mathbf{A l}_{\mathbf{x}} \mathbf{G a}_{(1-x)} \mathbf{A s}$ \\
\hline$p$ - confinement $A \mathbf{I}_{x} \mid \mathbf{n}_{(1-x)} P$ \\
\hline MQW \\
\hline$n$ - confinement $A \mathbf{I}_{x} \operatorname{In}_{(1-x)} P$ \\
\hline $\mathrm{n}$ - contact layer $\left(\mathrm{Al}_{\mathrm{x}} \mathrm{Ga}_{(1-\mathrm{x})}\right)_{0.5} \mathrm{In}_{0.5} \mathrm{P}$ \\
\hline $\begin{array}{l}\text { GaAs Buffer layer } \\
\text { Substrate: } n \text { - GaAs }\end{array}$ \\
\hline
\end{tabular}

Figure 1: Schematic layer structure of high power red LED.

\section{EXPERIMENTAL DETAILS}

The optical parameters of the device under test were measured in both continuous wave $(\mathrm{CW})$ and pulsed regime. A "CDS600 " spectrometer from Labsphere Co, Ltd. was used to collect light output along with the "LightMtrx" software to record the radiometric and photometric quantities. A "Keithley 2400 " source was used to drive the LED under CW regime. Under pulsed regime, for currents up to $2 \mathrm{~A}$, an "Agilent $8114 \mathrm{~A}$ " pulse generator was used capable of producing pulses as short 
as $200 \mathrm{~ns}$. For the current range of 2-23 A and sub-microsecond pulse width, we utilized IXYS Colorado DEI generator with external triggering. For the sub-nanosecond high current (20-45 A) pulses, a specially designed high power handmade generator was used. The pulse width and duty cycle were monitored on the oscilloscope using a current probe. To measure the sub-nanosecond electroluminescence (EL) spectra an ultrafast photo detector of $30 \mathrm{GHz}$ bandwidth (BW) was used together with an Agilent 86100A $50 \mathrm{GHz}$ oscilloscope. And, Si-based photo detector with the $14 \mathrm{~ns}$ rise time was used for sub-microsecond measurement. The time-resolved response was monitored and recorded on the oscilloscope with BW of $1 \mathrm{GHz}$. The duty cycle was chosen in such a way, as to avoid the thermal impact of the previous pulses on the current one. Also, a closed-cycle Helium cryostat "Janis-CCS450" was used to perform temperature-dependent electroluminescense (TDEL) measurements.

\section{RESULTS AND DISCUSSION}

\subsection{Spectral behaviour}

Figure 2a demonstrates EL spectral dependence on pulsed current input. The pulsed input is varied over a broad current range from $100 \mathrm{~mA}$ to $5 \mathrm{~A}$ at a constant pulse duration of $100 \mu \mathrm{s}$ and $1 \%$ duty cycle. With increasing current, the emission peak shifts towards longer wavelengths, i.e. a redshift is observed ${ }^{17}$. This behaviour is similar to the behaviour observed under $\mathrm{CW}$ regime with the redshift starting at currents $\geq 10 \mathrm{~mA}$ and greatly enhanced at currents $>100 \mathrm{~mA}$.
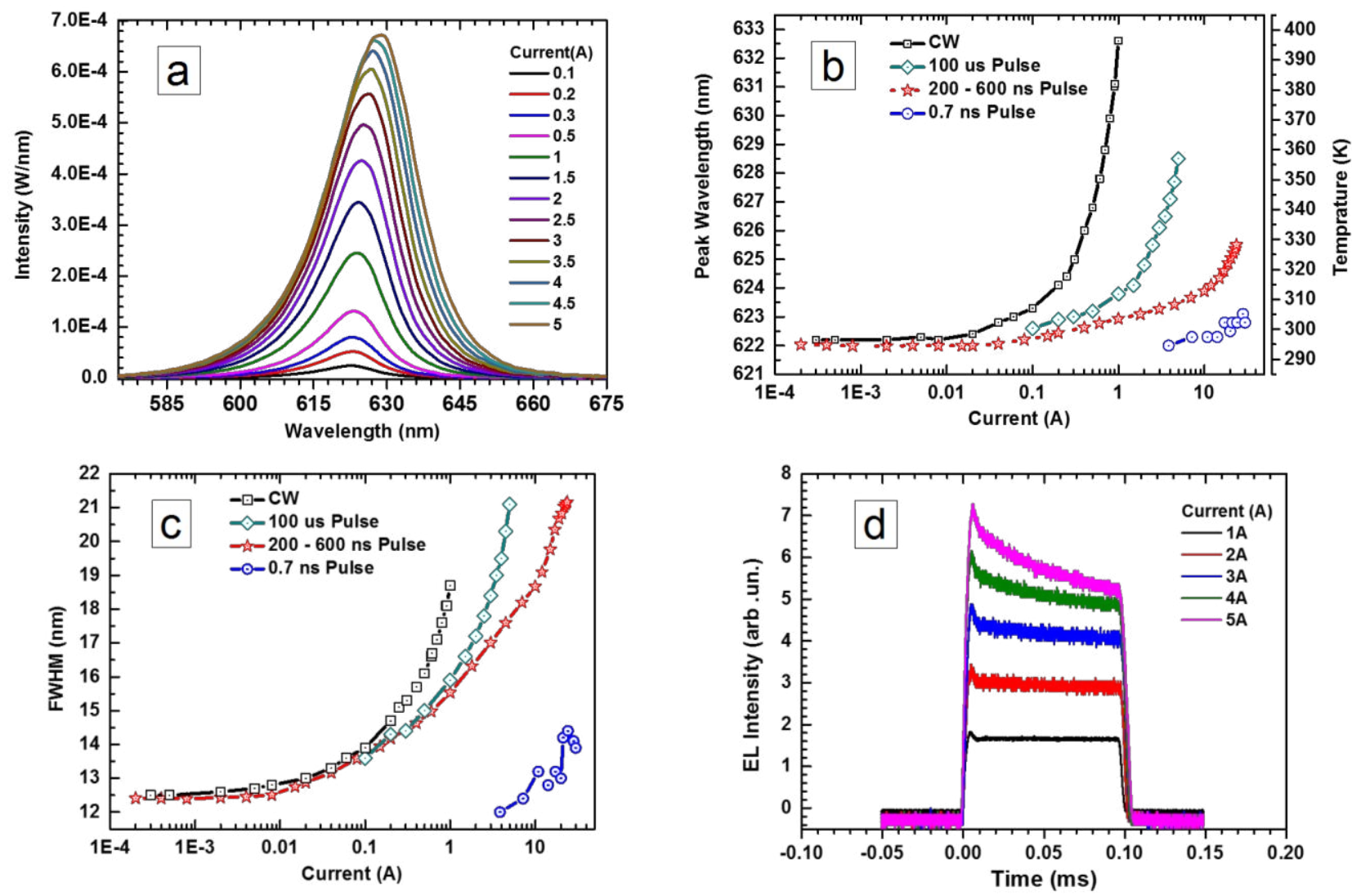

Figure 2: EL emission spectra of a red LED pumped by $100 \mu$ s pulses with $1 \%$ duty cycle in the current range from $100 \mathrm{~mA}$ to $1 \mathrm{~A}$ (a). Evolution of peak wavelength/corresponding temperature variation (b) and FWHM of the emission spectrum (c) with current at $\mathrm{CW}$ (squares) and $100 \mu \mathrm{s}$ (rhombus), 200-600 ns (stars), and $0.7 \mathrm{~ns}$ pulsed excitation (circles). A short-term, i.e. during-the-pulse EL intensity reduction observed at $100 \mu \mathrm{s}$ pulsed excitation for current magnitudes from $1 \mathrm{~A}$ to $5 \mathrm{~A}$. 
Under $100 \mu$ s pulse regime at driving currents up to $1 \mathrm{~A}$, a peak wavelength shift of $\sim 1 \mathrm{~nm}$ with a $\sim 2.5 \mathrm{~nm}$ increase in the spectrum full width half maximum (FWHM) is observed, as shown in Fig.2b,c, suggesting insignificant heating of the active region. However, at further increase in current up to $5 \mathrm{~A}$, a red shift of $\sim 6 \mathrm{~nm}$ (Fig.2b) and a spectral broadening of $\sim 28.5 \mathrm{~nm}$ (Fig.2c) present a strong evidence for elevated active region temperature.

The asymmetric shapes and the slope variation of the short-wavelength edge of the emission spectrum with current shown in Fig.2a, can be attributed to increasing junction temperature ${ }^{13}$. To further investigate into behaviour of the peak wavelength and spectral width, the EL intensity evolution during the microsecond current pulses was monitored via photodiode response under the same operating conditions, as shown in Fig.2d. To determine EQE, in this case, radiant flux was collected for time much longer than the pulse period and then normalised to the duty cycle. A decrease in the EL intensity during the pulse for more than $2 \mathrm{~A}$ of the current magnitude is evident

After the above observations with microsecond pulses, spectral redshift under sub-microsecond ( 100-600 ns) and subnanosecond $(0.7 \mathrm{~ns})$ pulsed pumping in the extended range of currents up to $23 \mathrm{~A}$ was investigated.

Also, as shown in figure $2 \mathrm{~b}$ and $2 \mathrm{c}$ respectively a redshift on $\sim 4 \mathrm{~nm}$ in peak wavelength and $\sim 10 \mathrm{~nm}$ of FWHM broadening is observed. Both the onset of decrease in efficiency and peak wavelngth redshift starts in the current range of 600-700 $\mathrm{mA}$. To understand temprature dependent shift of peak wavelength TDEL measurments over a wide temperature range from 13 to $450 \mathrm{~K}$ under $\mathrm{CW}$ regime at various currents were done. The experimantal dependence (peak wavelenght vs. temperature) was best described by a liner function with a coefficient of about $7.5 \mathrm{~K} / \mathrm{nm}$ for tempratures above $150 \mathrm{~K}$. Thus suggesting self-heating with change in temprature $\Delta \mathrm{T}$ was about $30 \mathrm{~K}$ corresponding to $4 \mathrm{~nm}$ red shift in peak wavelength.

It is seen from Fig. $2 \mathrm{~b}$ that LED pumping by sub-nanosecond ( $0.7 \mathrm{~ns})$ current pulses with the $0.1 \%$ duty cycle provides the redshift of $\sim 1 \mathrm{~nm}$ only. This suggests that the active region of the LED has negligible overheating under these pumping conditions.

\subsection{Efficiency vs. current analysis}
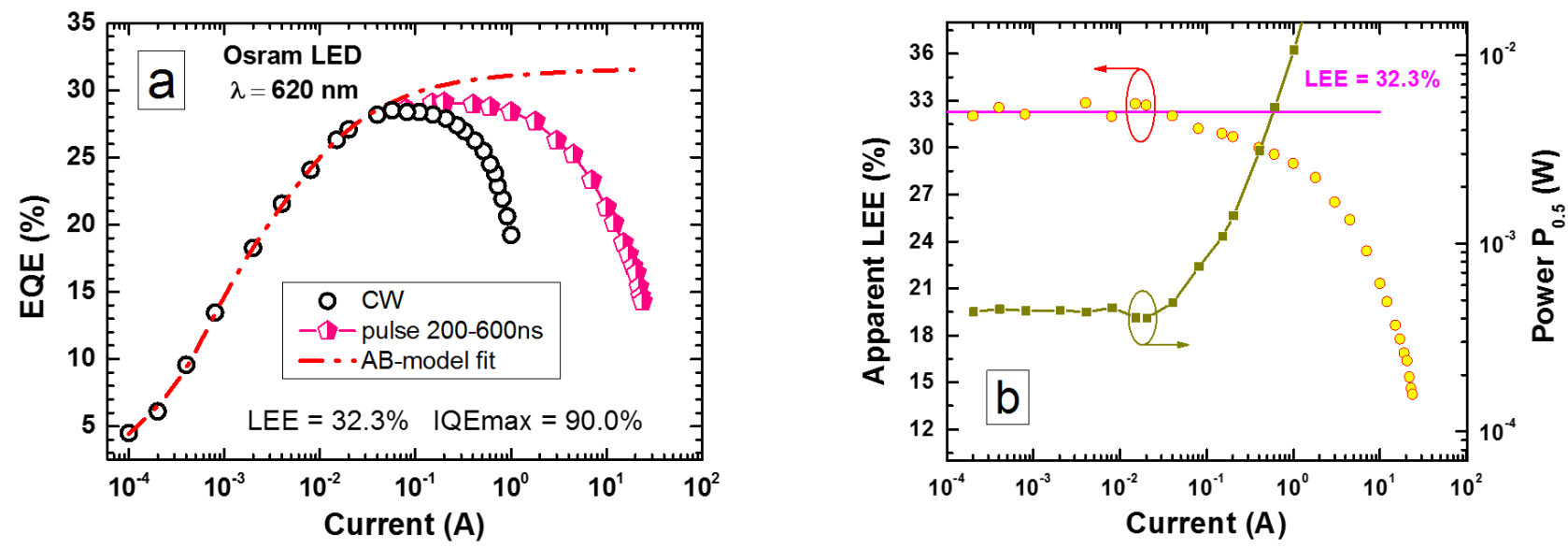

Figure 3: (a) External quantum efficiency under sub-microsecond pulses (half-filled pentagons) and CW regime (black open circles). Efficiency decrease is present for sub-microsecond pulses for current $>1 \mathrm{~A}$ after it starts to saturate beyond $100 \mathrm{~mA}$ of current. EQE is fitted with $\mathrm{AB}$ model (reduced $\mathrm{ABC}$ model) neglecting Auger coefficient, (b) apparent LEE and $P_{0.5}$ vs. current evaluated from experimental EQE data.

In order to estimate CW light extraction efficiency (LEE) and IQE of blue LEDs from the measured EQE(I) dependence, a special procedure based on the $\mathrm{ABC}$ recombination model has been suggested elsewhere ${ }^{18}$. Direct application of the procedure to red LEDs fails because of (i) small contribution of Auger recombination to the total recombination rate in the range of the current densities of interest and (ii) interfering the recombination processes with the electron leakage producing the efficiency decrease at high currents. Therefore, we suggest here an alternative procedure based on the reduced ABC-model called, hereafter, the AB-model. According to the model, only the Shockley-Read-Hall non-radiative recombination with the recombination constant $A$ and radiative recombination with the recombination constant $B$ are 
accounted for, assuming the 100\%-injection efficiency at low currents. Then EQE $\left(\eta_{e}\right)$ of an LED structure can be estimated from the equation

$$
\eta_{e}=\frac{\eta_{\text {ext }} P_{o u t}^{1 / 2}}{P_{0.5}^{1 / 2}+P_{o u t}^{1 / 2}}
$$

where $P_{\text {out }}=\hbar \omega \eta_{\text {ext }} V_{r} B n^{2}$ is the output optical power of the LED with the active region volume $V_{r}$ and non-equilibrium electron/hole concentration $n$; $\hbar \omega$ is the mean energy of the emitted photons and $\eta_{e x t}$ is LEE. In Eq.(1), $P_{0.5}=\hbar \omega \eta_{e x t} V_{r} A^{2} B^{-1}$ is the optical power corresponding to IQE of 50\%. This parameter is generally unknown and cannot be directly evaluated from the measured EQE(I) dependence. Nevertheless, it can be excluded by regarding any pair of experimental points from the dependence, giving

$$
\eta_{\text {ext }}=\frac{P_{2}^{1 / 2}-P_{1}^{1 / 2}}{P_{2}^{1 / 2} / \eta_{2}-P_{1}^{1 / 2} / \eta_{1}} \quad, \quad P_{0.5}^{1 / 2}=\frac{\eta_{2}-\eta_{1}}{\eta_{1} / P_{1}^{1 / 2}-\eta_{2} / P_{2}^{1 / 2}},
$$

where $\eta_{j}=\eta_{e}\left(P_{j}\right), j=1,2$ and $P_{j}$ being the output optical power of the LED measured at two different driving currents. So, regarding a measured point as the reference one we can obtain $\eta_{e x t}$ and $P_{0.5}$ as a function of current similar to that shown in Fig.3b. One can see from the figure that both parameters calculated by Eqs.(2) remain to be nearly constant and equal to $\eta_{\text {ext }}=32.3 \%$ and $P_{0.5}=0.45 \mathrm{~mW}$ at the currents less than $\sim 50-60 \mathrm{~mA}$. This value of $\eta_{e x t}$ is accosiated with the real LEE. At higher currents, apparent $\eta_{e x t}$ and $P_{0.5}$ estimated by Eqs.(2) start to vary, which is the evidence for IQE degradation caused by the device overheating or/and electron leakage.

Figure 3a shows the $\mathrm{EQE}(\mathrm{I})$ under $\mathrm{CW}$ and sub-microsecond excitation measured in an integrating sphere. Under $\mathrm{CW}$ regime of operation, the EQE rises steadily at lower current densities before it reaches it's maximum at $\sim 60 \mathrm{~mA}$. A decrease in efficiency is observed when pumped at currents $\geq 150 \mathrm{~mA}$. At the current of $700 \mathrm{~mA}, \mathrm{EQE}$ drops by $15 \%$ of its maximum value. This sharp decrease in the efficeincy can be expained by device self-heating. Unlike CW regime, the maximum efficiency is approached at the current of $\sim 200-250 \mathrm{~mA}$ at the sub-microsecond pulsed excitation, and the $15 \%$ efficiency drop is observed at $\sim 8-9 \mathrm{~A}$.

The dash line on the Fig.3a shows EQE(I) predicted by the reduced AB-model, assuming isothermal operation conditions and no electron leakage to occur. Comparison of its behavior with the measured CW EQE(I) dependences enables estimation of the maximum IQE obtained at the curents of $\sim 0.2-0.5 \mathrm{~A}$, which is of $\sim 90.0 \%$ and compares well with the state-of-the-art. The low EQE maximum of $\sim 29 \%$ is a result of a relatively low LEE $=32.3 \%$ specific for the devices under investigation that do not feature the typical lens cover. The AB-model fits very well to the experimental data of the low current $\mathrm{CW}$ branch, neglecting Auger recombination.

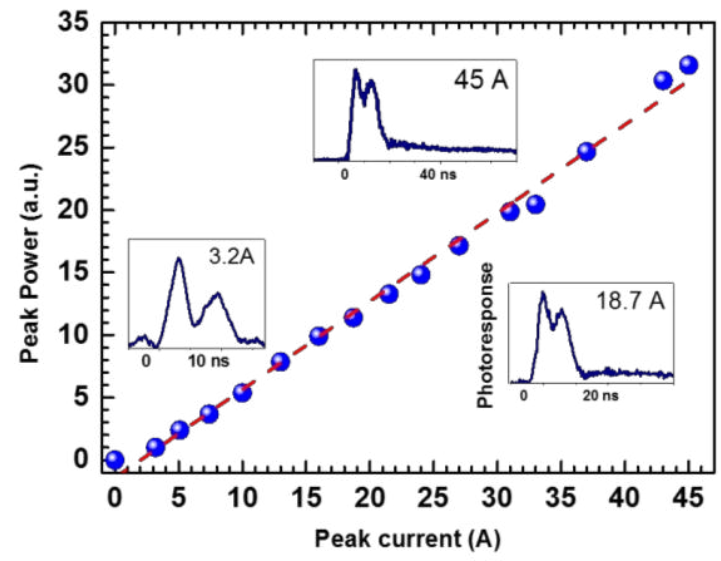

Figure 4: Peak power behavior under wide range of $0.7 \mathrm{~ns}$ peak current up to $45 \mathrm{~A}$ with $1 \%$ duty cycle, where blue circles represent the data and linear fitting is depicted by the dashed red line. In inset the photo-response pulse at 5, 20 and $45 \mathrm{~A}$ as observed on the oscilloscope is shown. 
In order to exclude the effect of self-heating in our experiments, we studied the photo-response of LEDs under a wide range of $0.7 \mathrm{~ns}$ pulsed current pumping up to $45 \mathrm{~A}$ with $0.1 \%$ duty cycle. Figure 4 shows the typical dependence of the maximum peak output power of LED on the peak current of the pumping pulse. The insets to Fig.4 show the time-traces of the LED output recorded by $30 \mathrm{GHz}$ BW photodetector and $50 \mathrm{GHz}$ oscilloscope. The observed second peak of smaller amplitude of the LED output can be explained by parasitic oscillations. The experimental data (blue circles) is well expressed by a linear fitting function (dashed red line). The observed linear dependence would imply EQE not to decrease at high currents. This conclusion should be regarded, however with some care. Preliminary simulations of the timedependent EL of the LED has shown that duration of the optical response is remarkably longer than that of the subnanosecond current pulse. This requires reconsidering the usual way of IQE and, hence, EQE determination, which will be done elsewhere. Nevertheless, the general conclusion could be done as following: the EQE does not decrease even at high currents, that means that increase in the carrier concentration in the active region without overheating does not result in the enhanced electron leakage and, hence, in the efficiency reduction.

\section{CONCLUSION}

To understand the nature of efficiency decrease at extremely high pump currents, the Golden Dragon-cased high power red LEDs were investigated under $\mathrm{CW}$ and pulsed regimes. Under $\mathrm{CW}$ operation the observed redshift of peak emission wavelength by $\sim 10 \mathrm{~nm}$ and spectral broadening of $\sim 4 \mathrm{~nm}$ for current range of $100 \mathrm{~mA}$ to $1 \mathrm{~A}$ is present due to device overheating. This is manifested on the EQE behavior as $10 \%$ decrease in efficiency. Further, as expected, a low LEE due to the absence of silicone lens, of $32.3 \%$ and maximum IQE of $90 \%$ was estimated using $\mathrm{AB}$ model based on $\mathrm{ABC}$ recombination model excluding the Auger coefficient. Pulses with duty cycle of $1 \%$ and wide range of durations from 100 $\mu$ s to sub-microsecond with increasing extended current range were applied to mitigate the effect of self-heating. For 100 $\mu$ s second pulses the observed photodiode response showed a decrease in intensity during the pulse, indicative of device self-heating at this pulse duration. EQE behavior similar to CW regime is observed for sub-microsecond (100 - $600 \mathrm{~ns})$ pulses. However, the onset of efficiency decrease it is shifted to higher current of $\geq 600 \mathrm{~mA}$ along with the redshift of peak wavelength. This shift of $4 \mathrm{~nm}$ corresponds to $30 \mathrm{~K}$ temperature increase. Thus a direct correlation between onset of the efficiency decrease and LED overheating is observed. To exclude the effect of self-heating sub-nanosecond pulses of 0.7 ns with duty cycle of $0.1 \%$ and up to $45 \mathrm{~A}$ of peak current were applied. A linearly dependence of LED peak output power on increasing peak current is observed indicating towards lowering or no decrease in EQE under this regime even at such high current pulsed pumping along with a red shift of $\leq 1 \mathrm{~nm}$. Nevertheless, it is observed that self-heating is an important factor contributing to efficiency decrease in these devices under all pumping regimes except for sub-nanosecond (0.7 ns) pulses. Since electron overflow is also temperature-dependent, we still cannot distinguish between the thermal effect on the competition between radiative and non-radiative recombination and electron overflow. The sub-nanosecond pumping would clarify this issue but this requires, at least, another way for EQE determination, as the duration of the optical response seems to be remarkably longer than the current pulse duration.

\section{ACKNOWLEDGMENTS}

This work was supported by European Union FP7, NEWLED project, Grant number 318388.

\section{REFERENCES}

[1] Vanderwater, D. A., Tan, I. H., Höfler, G. E., Defevere, D. C.., Kish, F. A., "High-brightness AlGaInP light emitting diodes," Proc. IEEE 85(11), 1752-1763 (1997).

[2] Tsao, J. Y., Crawford, M. H., Coltrin, M. E., Fischer, A. J., Koleske, D. D., Subramania, G. S., Wang, G. T., Wierer, J. J., Karlicek, R. F., "Toward Smart and Ultra-efficient Solid-State Lighting,” Adv. Opt. Mater. 2(9), 809836 (2014).

[3] Shi, J.-W., Kuo, F.-M., Lin, C.-W., Chen, W., Yan, L.-J.., Sheu, J.-K., "Investigation of the Efficiency-Droop Mechanism in Vertical Red Light-Emitting Diodes Using a Dynamic Measurement Technique," IEEE Photonics Technol. Lett. 23(21), 1585-1587 (2011).

[4] Meyaard, D. S., Shan, Q., Dai, Q., Cho, J., Schubert, E. F., Kim, M.-H., Sone, C., "On the temperature dependence 
of electron leakage from the active region of GaInN/GaN light-emitting diodes," Appl. Phys. Lett. 99(4), 041112 (2011).

[5] Xu, J., Schubert, M. F., Noemaun, A. N., Zhu, D., Kim, J. K., Schubert, E. F., Kim, M. H., Chung, H. J., Yoon, S., et al., "Reduction in efficiency droop, forward voltage, ideality factor, and wavelength shift in polarizationmatched GaInN/GaInN multi-quantum-well light-emitting diodes,” Appl. Phys. Lett. 94(1), 011113 (2009).

[6] Jong-In, S., Hyungsung, K., Dong-Soo, S., Han-Youl, Y., "An Explanation of Efficiency Droop in InGaN-based Light Emitting Diodes: Saturated Radiative Recombination Rate at Randomly Distributed In-Rich Active Areas," J. Korean Phys. Soc. 58(3), 503 (2011).

[7] Shen, Y. C., Mueller, G. O., Watanabe, S., Gardner, N. F., Munkholm, A., Krames, M. R., "Auger recombination in InGaN measured by photoluminescence," Appl. Phys. Lett. 91(14), 141101 (2007).

[8] Delaney, K. T., Rinke, P., Van de Walle, C. G., "Auger recombination rates in nitrides from first principles," Appl. Phys. Lett. 94(19), 191109 (2009).

[9] Mukai, T., Yamada, M., Nakamura, S., "Characteristics of InGaN-Based UV/Blue/Green/Amber/Red LightEmitting Diodes,” Jpn. J. Appl. Phys. 38(Part 1, No. 7A), 3976-3981 (1999).

[10] Bochkareva, N. I., Voronenkov, V. V., Gorbunov, R. I., Zubrilov, A. S., Lelikov, Y. S., Latyshev, P. E., Rebane, Y. T., Tsyuk, A. I., Shreter, Y. G., "Defect-related tunneling mechanism of efficiency droop in III-nitride lightemitting diodes," Appl. Phys. Lett. 96(13), 133502 (2010).

[11] Cho, J., Schubert, E. F., Kim, J. K., "Efficiency droop in light-emitting diodes: Challenges and countermeasures," Laser Photon. Rev. 7(3), 408-421 (2013).

[12] Huang, C.-F., Su, Y.-F., Lin, C.-B.., Chiang, K.-N., "Research on efficiency droop mechanism and improvement in AlGaInP Ultra-High-Brightness LEDs using the transient measurement method," Solid. State. Electron. 93, 1520, Elsevier Ltd (2014).

[13] Shim, J., Han, D., Kim, H., Shin, D., Lin, G., Meyaard, D. S., Shan, Q., Cho, J., Fred Schubert, E., et al., "Efficiency droop in AlGaInP and GaInN light-emitting diodes," Appl. Phys. Lett. 100(11), 111106 (2012).

[14] Royo, P., Stanley, R. P., Ilegems, M., Streubel, K.., Gulden, K. H., "Experimental determination of the internal quantum efficiency of AlGaInP microcavity light-emitting diodes," J. Appl. Phys. 91(5), 2563 (2002).

[15] Windisch, R., Altieri, P., Butendeich, R., Illek, S., Stauss, P., Stein, W., Wegleiter, W., Wirth, R., Zull, H., et al., "InGaAlP thin film LEDs with high luminous efficiency," Proc. SPIE 5366, 43-52 (2004).

[16] Peter, M., Laubsch, A., Bergbauer, W., Meyer, T., Sabathil, M., Baur, J., Hahn, B., "New developments in green LEDs,” Phys. Status Solidi Appl. Mater. Sci. 206(6), 1125-1129 (2009).

[17] Manninen, P., Orreveteläinen, P., "On spectral and thermal behaviors of AlGaInP light-emitting diodes under pulse-width modulation,” Appl. Phys. Lett. 91(18), 17-20 (2007).

[18] Titkov, I. E., Karpov, S. Y., Yadav, A., Zerova, V. L., Zulonas, M., Galler, B., Strassburg, M., Pietzonka, I., Lugauer, H.-J., et al., "Temperature-Dependent Internal Quantum Efficiency of Blue High-Brightness LightEmitting Diodes,” IEEE J. Quantum Electron. 50(11), 911-920 (2014). 